\title{
Heat-resistant ohmic and barrier contacts for GaAs-based microwave devices
}

\author{
V.N. Ivanov' ${ }^{1}$ R.V. Konakova ${ }^{2}$, V.V. Milenin ${ }^{2}$, M.A. Stovpovoi ${ }^{1}$

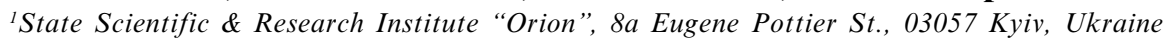 \\ ${ }^{2} V$. Lashkaryov Institute of Semiconductor Physics, NAS of Ukraine, 45 prospect Nauky, 03028 Kyiv, Ukraine \\ Phone: +38(044) 265 6182; fax: +38(044) 265 8342; e-mail: konakova@isp.kiev.ua
}

\begin{abstract}
We studied barrier and ohmic contacts that are used when developing GaAs-based microwave devices. It is shown that Au-Mo- $\mathrm{TiB}_{x}-n-\mathrm{GaAs}$ barrier contacts have higher thermal stability than Au-Ti-n-GaAs ones. This is due to substantial slowdown of grain-boundary diffusion in Au-Mo-TiB ${ }_{x}$ contacts resulting from use of amorphous $\mathrm{TiB}_{x}$ layers as barrier material. A phase transition in them occurs at a temperature over $1000{ }^{\circ} \mathrm{C}$. It was determined

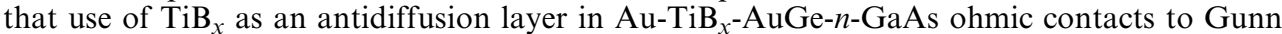
diodes increases their service life as compared to the traditional Au-AuGe- $n$-GaAs ohmic contact.
\end{abstract}

Keywords: gallium arsenide, ohmic contact, barrier contact, antidiffusion layer, mass transport.

Paper received 08.06.03; accepted for publication 17.06.03.

\section{Introduction}

At present the GaAs-based devices are widely applied in various areas of electronics, power and computer engineering, solar power engineering and microwave electronics. This set to the developers of active elements on the basis of GaAs and solid solutions strict requirements for tolerance of the element base used to different external actions, in particular, thermal ones [1-3]. The latter substantially affect both the properties of semiconductor material itself (up to changing the minority charge carrier lifetime and dopant compensation) and parameters of ohmic and barrier contacts. As a result, intense mass transfer occurs and interactions between phases appear that lead to degradation of the contact structures, as well as device as a whole. That is why the development of ohmic and rectifying contacts to GaAs with improved heat tolerance, high reproducibility and manufacturability still remains the urgent physico-technological challenge.

The objective of this work was to develop the required technology and study heat-resistant ohmic and barrier contacts that could be used in different GaAs-based devices.

\section{Sample preparation}

We used the epitaxial $n-n^{+}$-GaAs structures as starting semiconductor material. The epitaxial film thicknesses were $2.5-3 \mu \mathrm{m}$; the charge carrier concentration was (5-6) $\cdot 10^{15} \mathrm{~cm}^{-3}$. The structures intended for formation of ohmic (barrier) contacts were grown on semi-insulating (conductive) substrates. In both cases contact metallization was deposited onto the substrates using magnetron sputtering in argon. We used a modernized УBH-75P2 plant whose processing chamber was equipped with four magnetrons and a facility for pulse photon cleaning of sample surfaces. The processing chamber enabled one to deposit up to four different materials in any order, with preliminary cleaning of the epitaxial structure surface, in a unified technological cycle (without depressurization). After sample setting the chamber volume was evacuated with a high-vacuum molecular pump to the pressure of no higher than $6.5 \cdot 10^{-5} \mathrm{~Pa}$, with concurrent heating up to $60^{\circ} \mathrm{C}$. Then the chamber was cooled to room temperature and filled with argon up to a pressure of $2.7 \cdot 10^{-1} \mathrm{~Pa}$, and contact sputtering was performed.

The $\mathrm{Au}-\mathrm{TiB}_{x}$-AuGe system was used to form ohmic contacts. The layer thicknesses were $0.2,0.1$ and $0.03 \mu \mathrm{m}$, 
respectively. The mode of $\mathrm{TiB}_{x}$ sputtering was chosen in such a way as to form an amorphous film. After deposition of contact metallization, the samples were exposed to thermal treatment in hydrogen for $60 \mathrm{~s}$ at a temperature of $500^{\circ} \mathrm{C}$. Then the test structures to measure contact resistivity were fabricated using direct photolithography. The transmission line technique [2] was applied.

Both $\mathrm{Au}-\mathrm{Mo}-\mathrm{TiB}_{x}$ and $\mathrm{Au}-\mathrm{Ti}$ systems were used to form barrier contacts. The corresponding layer thicknesses were $0.2,0.05$ and $0.1 \mu \mathrm{m}$ and $0.1,0.05 \mu \mathrm{m}$, respectively. An Au-TiB ${ }_{x}$-AuGe ohmic contact was sputtered on the conductive substrate side. Test structures to take $I-V$ and $C-V$ curves of the barrier were made with direct photolithography.

To study heat resistance of ohmic and barrier contacts made, we applied rapid thermal annealing (RTA) at temperature $T=450^{\circ} \mathrm{C}$ for $60 \mathrm{~s}$ in hydrogen. Contact service life was tested by performing standardized tests of diode prototypes at package temperature of 87 and $100^{\circ} \mathrm{C}$

\section{Experimental results and discussion}

\subsection{Au-Ti(Mo-TiB $)$-GaAs barrier contacts}

Shown in Fig. 1 are the forward and reverse branches of $I-V$ curves for the Au-Ti- $n-n^{+}-\mathrm{GaAs}$ diode structures taken before (curves A, C) and after (curves B, D) RTA at $T=450{ }^{\circ} \mathrm{C}$ for $60 \mathrm{~s}$. One can see that the initial (before RTA) forward branch, as well as that after RTA, involve the following three sections: initial (from 0.025 to $0.25 \mathrm{~V}$ ), exponential (from 0.3 to $0.5 \mathrm{~V}$ ) and that at voltages over $0.5 \mathrm{~V}$. It was shown earlier in $[4,5]$ that such sections of the forward branch of $I-V$ curve are related to lateral nonuniformity of contact. This nonuniformity results in excess current at the initial section of $I-V$ curve before RTA and current increase after RTA. The exponential section is described by the following expression:

$I=I_{0} \exp \frac{q V}{n k T}$,

where

$I_{0}=A S T^{2} \exp \left(-\frac{q \varphi_{b}}{k T}\right)$,

$I$ is the current through the barrier; $I_{0}$ is the saturation current; $q$ is the elementary charge; $V$ is the applied voltage; $n$ is the ideality factor; $k$ is the Boltzmann constant; $A=8.4 \mathrm{~A} \cdot \mathrm{cm}^{-2} \cdot \mathrm{K}^{-2}$ is the Richardson constant for GaAs; $S$ is the barrier contact area; $\varphi_{b}$ is the Schottky barrier height.

It was found that RTA increased the Schottky barrier height by $40 \mathrm{meV}$ (from the initial value $\varphi_{b}=0.81 \mathrm{eV}$ to that after RTA $\varphi_{b}=0.85 \mathrm{eV}$ ). The ideality factor remained practically the same $(n=1.12)$. The third section of $I-V$ curve (related to the effect of base resistance on

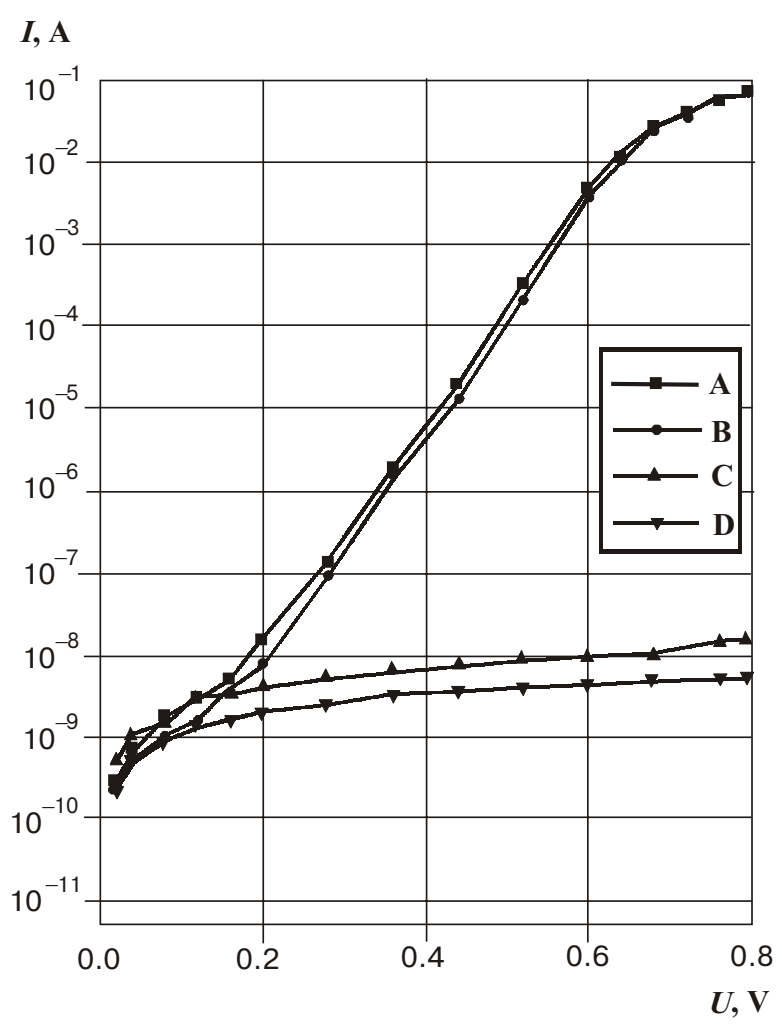

Fig. 1. $I-V$ curves for the Au-Ti- $n-n^{+}-\mathrm{GaAs}$ contacts; A and $\mathrm{C}-$ forward and reverse bias, respectively, for the initial sample; B and $\mathrm{D}$ - same for the sample after thermal treatment at $450{ }^{\circ} \mathrm{C}$ for $1 \mathrm{~min}$.

current flow) practically did not change after RTA, while the reverse current somewhat increased.

The above results can be explained if one takes into account the concentration depth profiles for the Au-Ti-n$n^{+}$-GaAs contact components taken before and after RTA (see Fig. 2). Indeed, in the initial sample (Fig. 2a) there is a rather expanded section of junction layer formed as a result of titanium mass transfer into gallium arsenide. After RTA this section is considerably expanded into $\mathrm{GaAs}$ and qualitatively changed due to RTA-induced $\mathrm{Au}$ penetration into the junction layer and GaAs near-contact region (Fig. 2b). Both above factors enhance lateral nonuniformity, due to increased (after RTA) Ti concentration near the boundary between phases, as well as to possible formation of new phases in the contacts, with predominance of gallium and titanium oxides and gold gallides.

Variations of structural-defect state in the GaAs nearcontact region led to appearance of excess currents at both forward and reverse branches of $I-V$ curve, the Schottky barrier parameters being practically unchanged. Many authors believe that just these factors (growth of the junction layer width and formation of new phases in the contact) are undesirable at formation of gate contacts in GaAs-based FETs and barrier contacts in GaAs-based IMPATT diodes [1-4]. 
C, at. $\%$

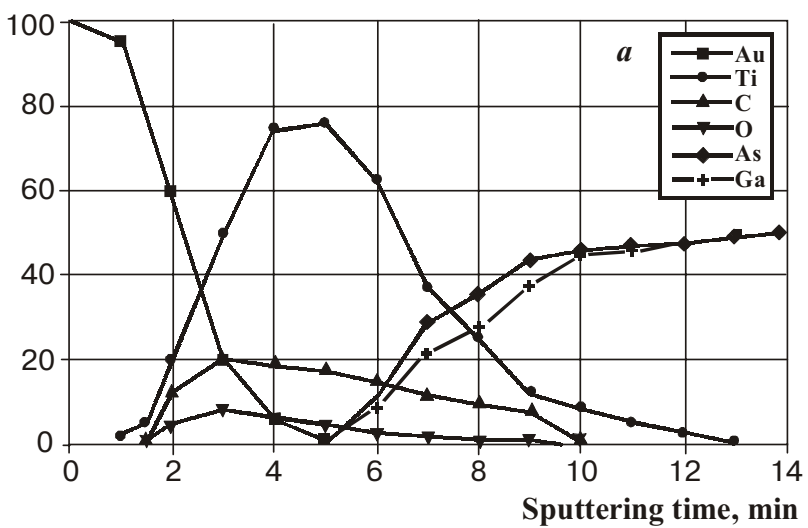

C, at. $\%$

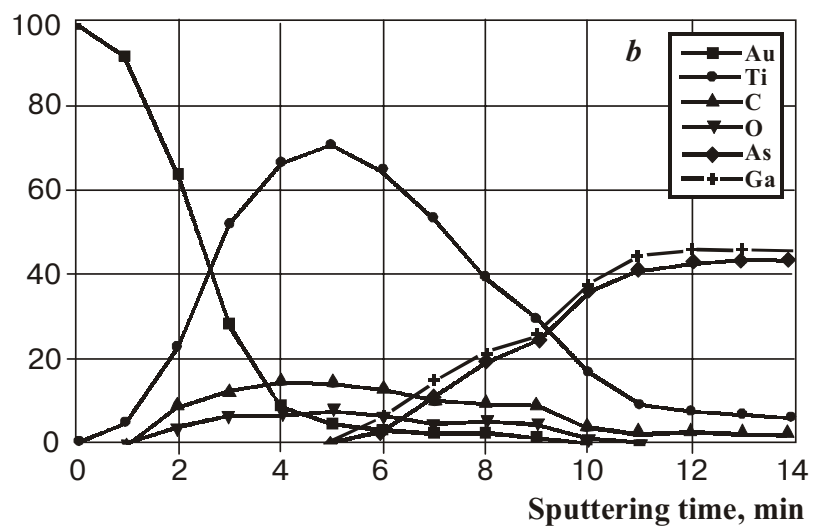

Fig. 2. Concentration depth profiles for the Au-Ti- $n-n^{+}-\mathrm{GaAs}$ contacts: $a$-initial sample, $b$ - sample after thermal treatment at $450{ }^{\circ} \mathrm{C}$ for $1 \mathrm{~min}$.

One can eliminate intense phase formation and mass transfer at the boundary between phases by $(i)$ placing an antidiffusion barrier between the gold film and barrierforming metal layer, and (ii) forming a rectifying contact by an amorphous titanium or zirconium diboride layer (and not by a polycrystalline film of a refractory metal), as was shown in our work [6]. We proposed to use the Au$\mathrm{Mo}^{-\mathrm{TiB}_{x}}$ structure as such construction of metallization. In this structure amorphous $\mathrm{TiB}_{x}$ serves as barrier-forming layer, while Mo serves as antidiffusion layer.

Shown in Fig. 3 are typical forward and reverse branches of $I-V$ curves for the Au-Mo-TiB ${ }_{x}$-GaAs Schottky diodes taken before (curves A, C) and after (curves B, D) RTA. One can see that, as in the case of the Au-Ti- $n-n^{+}-$ GaAs diode structures, the forward branches of $I-V$ curves involve three sections. However, the initial section in the forward branches of $I-V$ curve for the starting sample is less pronounced than that for the starting Au-Ti- $n-n^{+}-$ GaAs structures. In addition, after RTA the excess current at this section drops rather than grows, as is shown in Fig. 1. The exponential section for these diode struc-

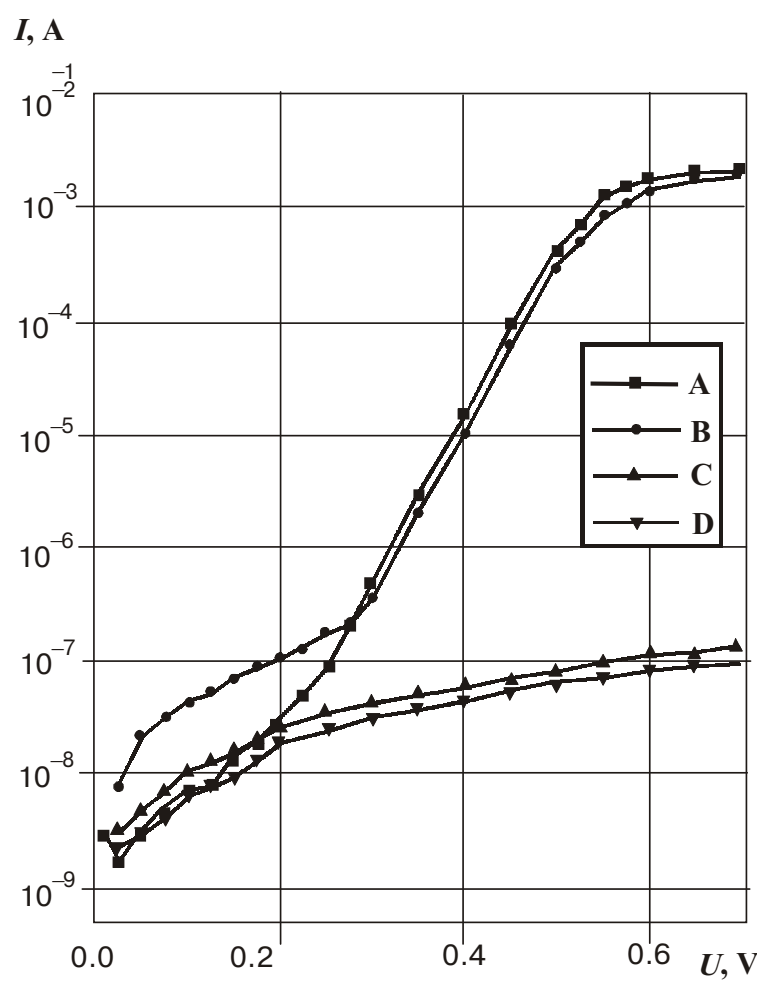

Fig. 3. As in Fig. 1, but for the Au-Mo-TiB $x^{-} n-n^{+}-\mathrm{GaAs}$ contacts.

tures is by 2.5 orders of magnitude more expanded than that for the Au-Ti- $n-n^{+}-$GaAs structures. The Schottky barrier height for the initial and annealed samples is 0.75 $076 \mathrm{eV}$, while the ideality factor $n \approx 1.08$. The third section in the forward branch of $I-V$ curve did not change after RTA, and the reverse branch even improved (the leakage current dropped).

The above results indicate at absence of considerable changes near the metal-semiconductor interface. This conclusion is confirmed by the concentration depth profiles for the Au-Mo-TiB $x_{x}-n-n^{+}$-GaAs structure components, as well as by the gold surface morphology in contacts before and after RTA (see Figs 4 and 5). Indeed, one can see from Figs $5 a, b$ that the layer separating gold from $\mathrm{TiB}_{x}$ is characterized by low Au diffusion coefficient and good adhesion properties. These facts enable to state that no considerable structural and chemical transformations occur in the antidiffusion layer. As to the barrier-forming $\mathrm{TiB}_{x}$ contact, one can see from Fig. $4 b$ that the junction layer at the $\mathrm{TiB}_{x} / n-n^{+}-\mathrm{GaAs}$ interface remains practically the same after RTA. A decrease of leakage current in $I-V$ curves serves as additional indication at some structural-impurity ordering in the near-contact region. 
C, at. \%

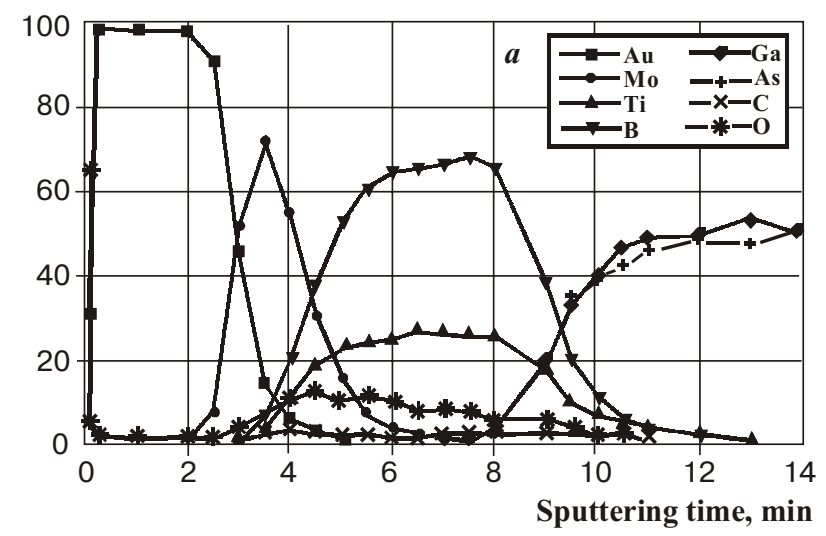

C, at. \%

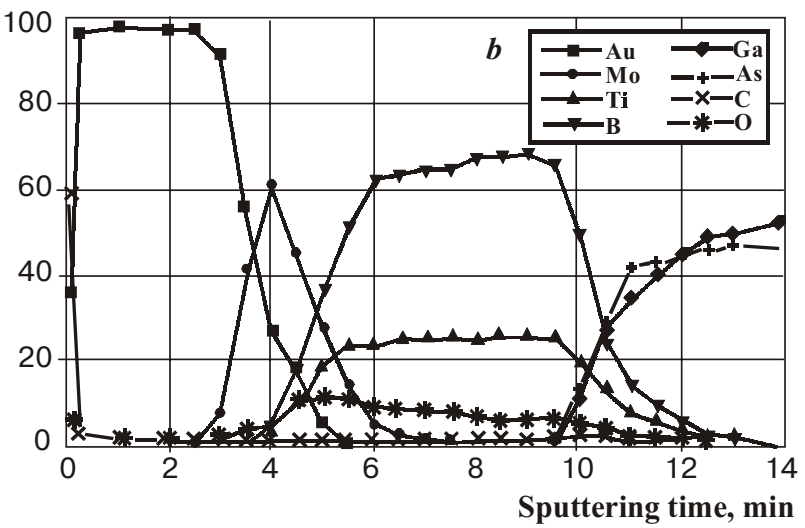

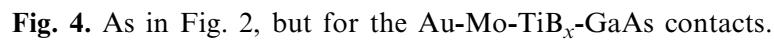
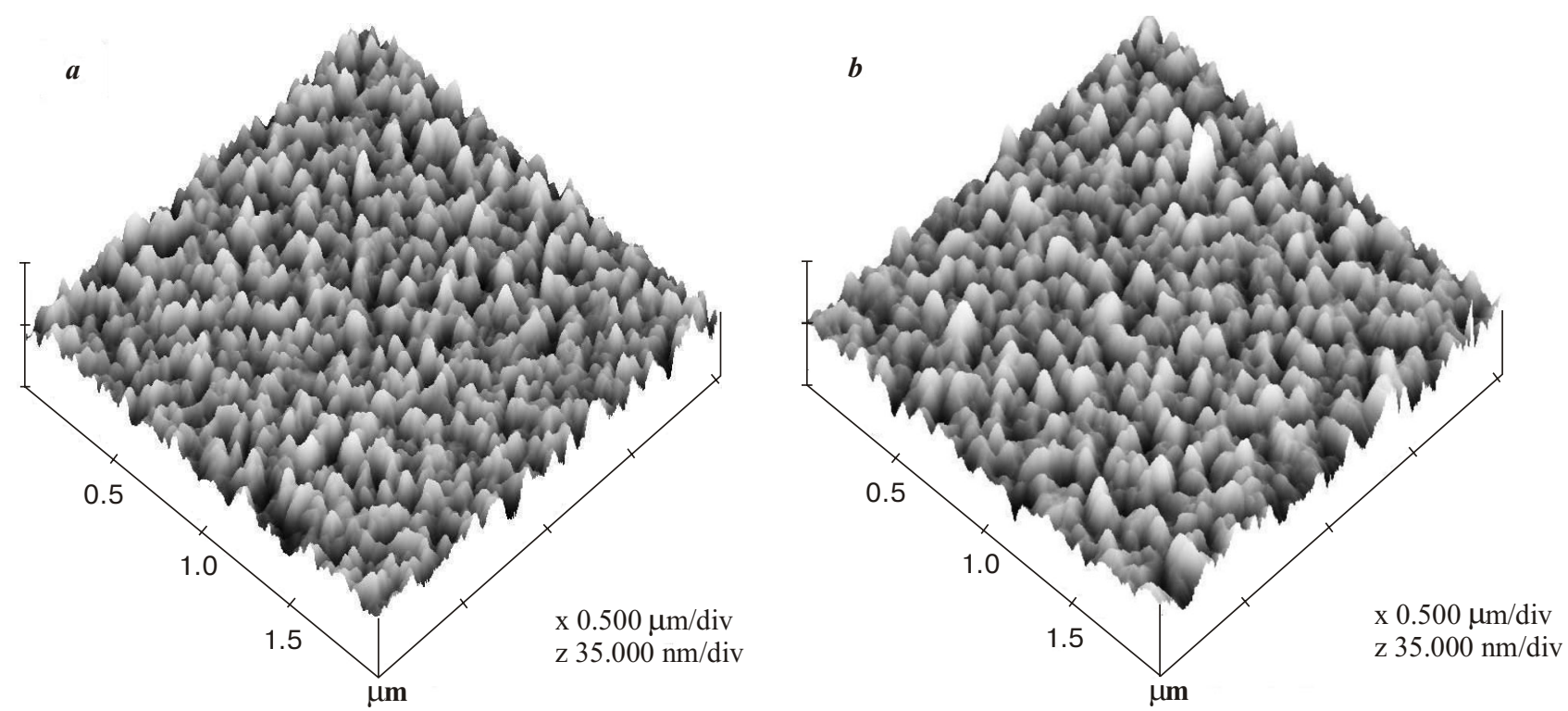

Fig. 5. Au film surface morphology for the Au-Mo-TiB $x_{x}-n-n^{+}-\mathrm{GaAs}$ contacts; $a$ - initial sample, $b$ - sample after thermal treatment at $450{ }^{\circ} \mathrm{C}$ for $1 \mathrm{~min}$.

\section{2. $A u-T i B_{x}-A u G e-G a A s$ ohmic contacts}

Shown in Fig. 6 are the concentration depth profiles for the $\mathrm{Au}-\mathrm{TiB}_{x}-\mathrm{AuGe}-n-n^{+}-\mathrm{GaAs}$ contact components taken before and after RTA. One can see that the $\mathrm{TiB}_{x}$ layer is antidiffusion (as in the previous case). This property is displayed by absence of interaction between gold and the upper metallization layer, as well as ohmic contact. Resistivity of ohmic contacts of this type formed in test structures was about $10^{-5} \Omega \cdot \mathrm{cm}^{2}$ (both before and after RTA). This is in good agreement with the results of [6].

The above results also correlate well with resistivity of the ohmic contacts to Gunn diodes (measured before and after RTA), as well as with the results of tests for service life of Gunn diodes (Fig. 7) with the Au-TiB $x^{-}$ AuGe- $n-n^{+}-n^{++}$-GaAs contacts performed at diode package temperature of 87 and $100{ }^{\circ} \mathrm{C}$ (Figs $7 a$ and $7 b$, respectively). One can see that, whatever the diode package temperature, contact resistivity $\rho_{c}$ remains practically the same during up to 10000 hours of diode operation. At the same time measurements of $\rho_{c}$ for Gunn diodes with the Au-AuGe- $n^{+}-n-n^{+}-n^{++}$-GaAs contacts at package temperature of $87^{\circ} \mathrm{C}$ have shown that after 500 hours of diode operation $\rho_{c}$ abruptly increased. This is related to the known substantial nonuniformity of such contacts [7]. 
C, at. $\%$

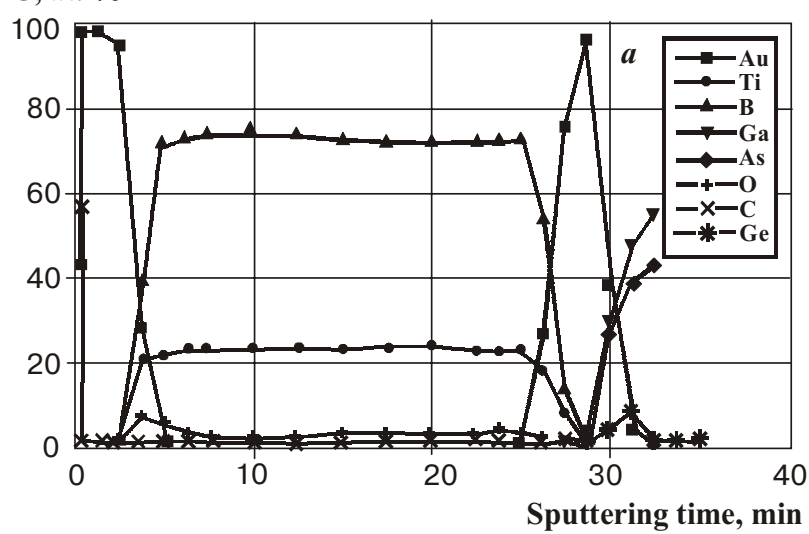

C, at. \%

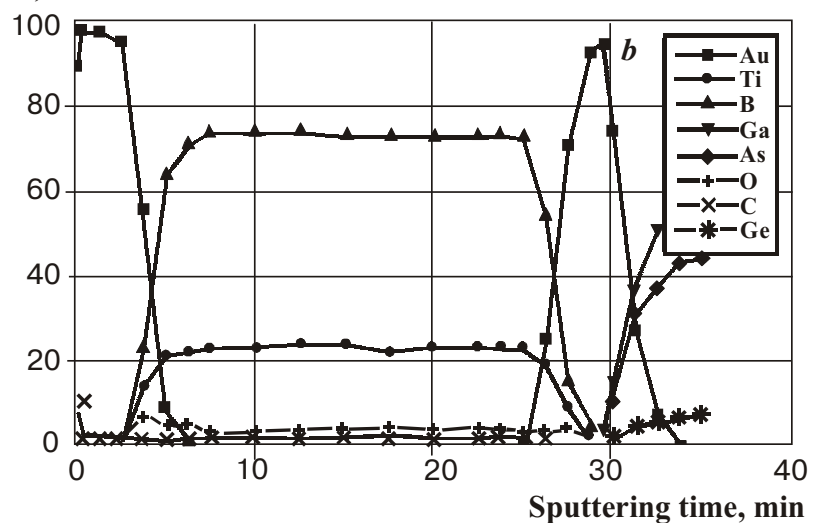

Fig. 6. As in Fig. 2, but for the Au-TiB $x^{-} \mathrm{AuGe}-n-n^{+}-i-\mathrm{GaAs}$ contacts.
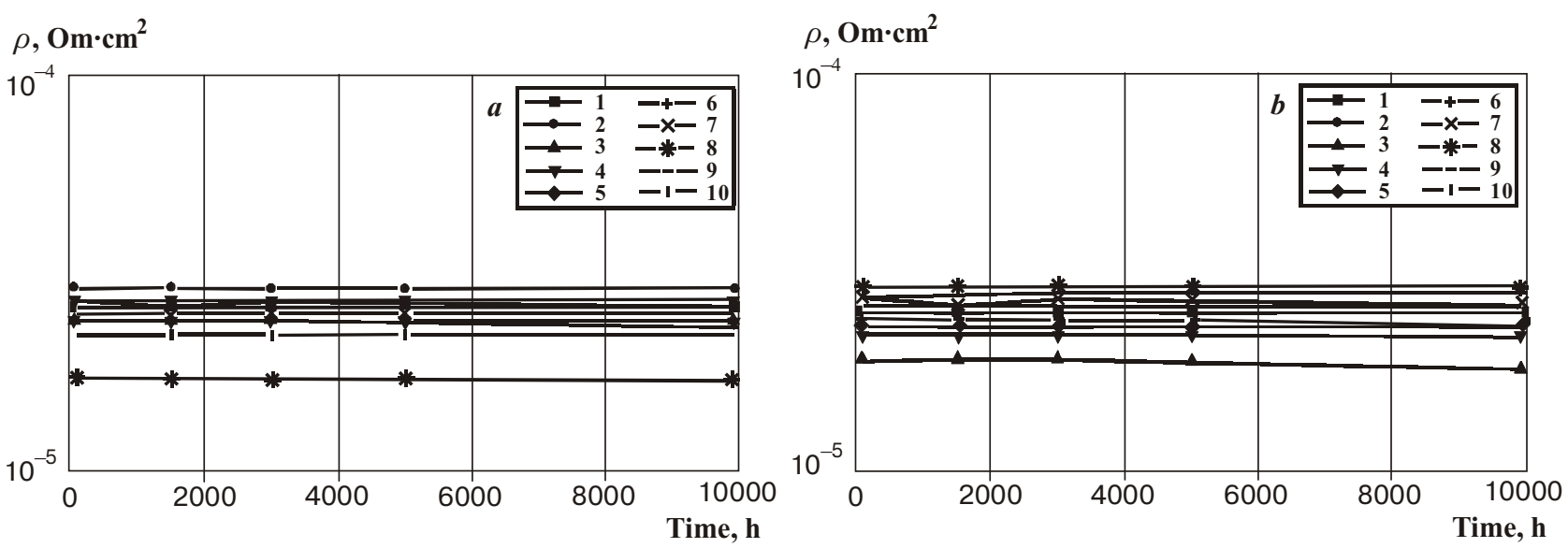

Fig. 7. Ohmic contact resistivity versus thermal treatment duration curves for the Au-TiB $x_{x}$ AuGe- $n-n^{+}-n^{++}-\mathrm{GaAs}$ Gunn diode contacts; $a(b)-$ at package temperature $T=87(100){ }^{\circ} \mathrm{C}$.

\section{Conclusions}

Our studies have shown that usage of amorphous $\mathrm{TiB}_{x}$ layers as barrier-forming and antidiffusion materials in multilayer contacts to GaAs makes it possible to advance a manufacturing technology for rectifying and ohmic contacts to GaAs that demonstrate steady operation for up to 10000 hours at package temperature of $87^{\circ} \mathrm{C}$.

\section{References}

1. VLSI Electronics: Microstructure Science, Eds. N.G. Einspruch, W.R. Wisseman, Vol.11 (GaAs Microelectronics), Academic Press, Orlando-San Diego-New York-London-TorontoMontreal-Sydney-Tokyo (1985).

2. M. Shur, GaAs Devices and Circuits, Plenum Press, New York and London (1987).
3. GaAs FET Principles and Technology, Eds. J.D. DiLorenzo, D.D. Khandelwal, Artech House, Inc. (1984).

4. E.F. Venger, R.V. Konakova, G.S. Korotchenkov, V.V. Milenin, E.V. Russu, I.V. Prokopenko, Interactions between Phases and Degradation Mechanisms in Metal-InP and Metal-GaAs Structures // Information-Editing Dept. of the Institute of Semiconductor Physics of the National Academy of Sciences of Ukraine, Kiev (1999) (in Russian).

5. V.I. Strikha, E.V. Buzaneva, Physical Foundations of the Metal-Semiconductor Contact Reliability in Integrated Electronics // Radio i Svyaz', Moscow (1987) (in Russian).

6. V.N. Ivanov, R.V. Konakova, V.V. Milenin, M.A. Stovpovoi, Contact-forming films of titanium borides and nitrides in gallium-arsenide microwave devices // Tekhnologiya i Konstruirovanie v Elektronnoi Apparature, No 6, pp. 54-56 (2002) (in Russian).

7. V.N. Chelyubeev, Effect of contact nonuniformities on the electrical characteristics of Gunn diodes // Synopsis of the Candidate of Technical Sci. Thesis, Kiev, Kiev Polytechnic Institute, 2002 (in Russian). 